pulmonary or muscular involvement. While the true frequency of heart block associated with anti-U1RNP remains to be determined, this study raises the consideration of echocardiographic surveillance in this setting. Women with MCTD should receive a specific counselling when planning a pregnancy, as it is currently done in women with SLE.

Disclosure of Interest: None declared

DOI: 10.1136/annrheumdis-2018-eular.5188

\section{THU0636 CANAKINUMAB TREATMENT IN ADULT-ONSET STILL'S DISEASE: CASE SERIES}

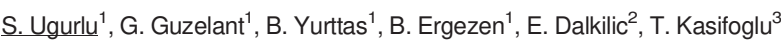
B. Yagiz ${ }^{2}$, H. Ozdogan ${ }^{1} .{ }^{1}$ Division of Rheumatology, Department of Internal Medicine, Istanbul University, Cerrahpasa Medical Faculty, Istanbul; ${ }^{2}$ Division of Rheumatology, Department of Internal Medicine, Uludag University, Medical Faculty, Bursa; ${ }^{3}$ Division of Rheumatology, Department of Internal Medicine, Osmangazi University, Medical Faculty, Eskisehir, Turkey

Background: In Adult-onset Still's disease (AOSD), cases refractory to typical DMARDs, Canakinumab (an anti-IL-1ß monoclonal antibody) has been reported to be effective in a limited number of refractory cases. ${ }^{1}$

Objectives: The aim of this retrospective study was to represent AOSD patients treated with Canakinumab in 3 centres.

Methods: The follow up data of 10 AOSD patients ( 8 female, 2 male), who were followed up in outpatient clinics of 3 tertiary centres were reviewed retrospectively. The initial characteristics and follow up findings were reported

Results: The mean timespan between the initial diagnosis and Canakinumab treatment $45.2 \pm 29$ months (mean $\pm S D$ ). Before the onset of Canakinumab therapy, all patients were exposed to methotrexate, 1 to leflunomide, 8 to Tocilizumab and 8 to Anakinra. As for the biologic agents, 3 patients were also treated beforehand with Infliximab, 2 with Adalimumab, 2 with Etanercept and 2 with Rituximab. Canakinumab therapy was initiated in all patients with the indication of refractory disease under other medications, except for the one in whom neutropenia became evident under anakinra. The mean number of Canakinumab injections was $9.3 \pm 8$. The mean follow-up period of patients treated with Canakinumab was $43.1 \pm 33$ months. Seven out of 10 patients are still being treated with Canakinumab of $150 \mathrm{mg} / \mathrm{month}$ and one of $150 \mathrm{mg} /$ every 2 months. One patient had a single injection and was fully controlled. The mean ferritin measure of 9 patients was reduced from $1292.3 \pm 1530 \mathrm{ng} / \mathrm{ml}$ to $354 \pm 530.2 \mathrm{ng} / \mathrm{ml}$ following the Canakinumab therapy $(p=0.035)$. The mean of patient-reported global visual analogue scale (PG-VAS) scores was reduced from $7.4 \pm 2.4$ to $2.3 \pm 2.2$ with Canakinumab $(p<0.001)$. Mean Erythrocyte sedimentation rate (ESR) was reduced from 44.2 \pm 35.1 to $22.7 \pm 26.5$ with the help of Canakinumab therapy $(p=0.005)$. Six patients are still on prednisolone at a maximum dose of $10 \mathrm{mg} /$ day. The indication of therapy termination in the remaining 1 patient was the diagnosis of tuberculosis at 9 th month of the treatment despite isoniazid prophylaxis. The patient was also treated with multiple biological agents beforehand, therefore it is not easy to conclude that treatment with Canakinumab induces tuberculosis flares.

Conclusions: Canakinumab treatment seems to be effective in refractory AOSD patients who were previously treated with various agents. We state that an IL-1 blocking agent, Canakinumab is a relatively safe and effective alternative in managing refractory AOSD cases. On the other hand, randomised controlled trials are needed to further investigate the role of Canakinumab in these cases as well as its use as the first choice of biologic agents.

\section{REFERENCE:}

[1] Kontzias A, Efthimiou P. The use of Canakinumab, a novel IL-1 $\beta$ long-acting inhibitor, in refractory adult-onset Still's disease. Semin Arthritis Rheum 2012;42(2):201-5.

Disclosure of Interest: None declared DOI: 10.1136/annrheumdis-2018-eular.7187

\section{EVALUATIONS OF COMPLEMENT PATHWAYS IN IGG4} RELATED DISEASE

S. Fukui, Y. Fujita, T. Origuchi, A. Kawakami. Immunology and Rheumatology, NAGASAKI UNIVERSITY GRADUATE SCHOOL OF BIOMEDICAL SCIENCES, Nagasaki, Japan

Background: In IgG4 related disease (IgG4-RD), hypocomplementemia is known to be seen. Complement pathways consist of three pathways, classical, alternative, and lectin pathways. Although $\lg$ G1 and $\lg G 3$ have ability to activate complement, IgG4 is known to be ineffective at activating complement.

Objectives: We attempted to elucidate which complement pathway is mainly associated with IgG4-RD.

Methods: Levels of complement elements and complement-associated elements, C1q, C2, C3, C3b/iC3b, C4, C4b, C5, C5a, C9, Factor D, Factor I, mannose-binding lectin (MBL), Factor $\mathrm{B}$, Factor $\mathrm{H}$, and properdin in preserved sera of patients with IgG4-RD at diagnosis and at remission were measured using multiplex bead-based assay. We compared complement levels at diagnosis of IgG4RD patients with those of sex-matched healthy donors.

Results: This study included 28 lgG4-RD patients and sex-matched 28 healthy donors. The median age at diagnosis and healthy donors' age were 65 [interquartile range (IQR): 55-70] and 64 (IQR:56-73), respectively. Patients with IgG4-RD at diagnosis had significantly higher levels of C5 and C5a $[33347 \mathrm{ng} / \mathrm{mL}$ vs $30375 \mathrm{ng} / \mathrm{mL}$ (median), $\mathrm{p}=0.0293,16417 \mathrm{pg} / \mathrm{mL}$ vs. $7083 \mathrm{pg} / \mathrm{mL}, \mathrm{p}<0.0001$ respectively] and significantly lower levels of C4, C4b, and Factor D (219671 ng/ $\mathrm{mL}$ vs. $325596 \mathrm{ng} / \mathrm{mL}, \mathrm{p}=0.0140,8784 \mathrm{ng} / \mathrm{mL}$ vs. $16285 \mathrm{ng} / \mathrm{mL}, \mathrm{p}=0.0101$ $4569 \mathrm{ng} / \mathrm{mL}$ vs. $5482 \mathrm{ng} / \mathrm{mL}, \mathrm{p}=0.0299$, respectively) compared to healthy donors. Levels of C5, C5a, C4, C4b, and Factor D were not different in two groups which were divided by clinical manifestations at diagnosis except for lower C4b levels in patients with lymphadenopathy compared with patients without lymphadenopathy. There were no differences in MBL, which was associated with the lectin pathway. In remission after the administrations of prednisolone, levels of $\mathrm{C} 5 \mathrm{a}$ significantly decreased compared to levels of C5a at diagnosis $(16305 \mathrm{pg} / \mathrm{mL}$ to $10029 \mathrm{pg} / \mathrm{mL}, \mathrm{p}=0.0043$ ). Other complement factors did not change significantly. Conclusions: The classical complement pathway may be associated with IgG4 $\mathrm{RD}$ rather than the alternative pathway and the lectin pathway based on results except for Factor D.

Disclosure of Interest: None declared

DOI: 10.1136/annrheumdis-2018-eular.5029

\section{THU0638 CLINICAL CHARACTERISTIC OF A GROUP OF PATIENTS WITH PSTPIP1-ASSOCIATED MYELOID- RELATED PROTEINEMIA INFLAMMATORY SYNDROME (PAMI)}

V.I. Burlakov ${ }^{1}$, A. Kozlova ${ }^{1}$, I. Mersiyanova ${ }^{2}$, A. Laberko $^{1}$, E. Viktorova $^{1}$, O. Shvets ${ }^{1}$, D. Yukhacheva ${ }^{1}$, G. Gordeeva ${ }^{1}$, E. Raykina ${ }^{2}$, V. Zakharova ${ }^{2}$

J. Rodina ${ }^{1}$, A. Shcherbina ${ }^{1}$. 'Immunology, National Medical Research Center of Pediatric Hematology, Oncology and Immunology named after Dmitry Rogachev, Moscow, Russia; ${ }^{2}$ Laboratory of Molecular Biology, National Medical Research Center of Pediatric Hematology, Oncology and Immunology named after Dmitry Rogachev, Moscow, Russian Federation

Background: PSTPIP1-gene associated autoinflammatory diseases is a group of clinically diverse syndromes predominantly manifested by various skin conditions (pyoderma gangrenosum, acne, hidradenitis suppurativa, necrotizing fasciitis). Yet one of them - PAMI - manifests mainly with haematologic abnormalities and autoinflammation with or without purulent features, presenting diagnostic difficulties for treating physicians - mainly haematologists. PAMI treatment is also challenging as IL-1 inhibitors alleviate inflammatory symptoms but cytopenias usually require additional therapeutic agents.

Methods: We describe five PAMI patients from 3 families: (2 girls, 2 boys, and affected mother of two patients). c.748G $>A(p . E 250 K)$ heterozygous mutation in PSTPIP1 was detected in all patients via targeted panel next generation sequencing and confirmed by Sanger sequencing.

Results: The median age of disease onset was 2 years (varied from at-birth onset to 7 years), the adult patient manifested at the age of 3 . As described all patients manifested with cytopenias: thrombocytopenia in three, severe anaemia in five neutropenia in three. All patients had elevated CRP and zinc levels, as previously described. Splenomegaly was noted in five, lymphadenopathy in one, colitis in one, severe arthritis in one (adult patient) and arthropathy in two patients. Mild pyodermia was noted only in one patient, two patients had skin vasculitis. One patient developed myelodisplastic syndrome and underwent successful hematopoietic stem cell transplantation (HSCT)

All patients received various immunosuppression prior to diagnosis with partial effect. After PAMI diagnosis rituximab was effective in two out of three, tocilizumab in one out of two, high dose anakinra in one patient. The girl who underwent HSCT is currently well, with full donor chimerism.

Conclusions: Haematologists need to be aware of the PAMI phenotype and include it in the diagnostic algorithm. Treatment still remains challenging and requires further investigation in larger groups of patients.

Disclosure of Interest: None declared

DOI: 10.1136/annrheumdis-2018-eular.7244 